\title{
Efficiency and effectiveness video on demand over worldwide interoperability for microwave access
}

\author{
Muthanna Jaafar Abbas ${ }^{1}$, Abeer Abd Al Hameed Mahmood ${ }^{2}$ \\ ${ }^{1}$ Department of Electrochemical Engineering, Babylon University, Iraq \\ ${ }^{2}$ Department of Electrical Engineering, Babylon University, Iraq
}

\section{Article Info \\ Article history: \\ Received Sep 26, 2018 \\ Revised Des 19, 2018 \\ Accepted Jan 11, 2019}

\section{Keywords:}

IPTV

QAM64

QPSK

VoD

WiMAX

\begin{abstract}
(WiMAX) is another solution for readiness broadband networks inside the region, covered or not, by alternative technologies like (ADSL) or cable through this paper by deploying many mobility patterns such as static, random and path for Video on Demand (VoD) over (WiMAX) Network. The performance of varied (QPSK) and (QAM) modulation techniques, by applying the (OPNET) simulator version 17.5. This work is finished in terms of the transmitted traffic and received traffic. Simulation result indicates that once nodes are moving by (SVC) code schema in (WiMAX). The result is an effective and attainable overall performance of (QPSK) which is better than (QAM) modulation technique.
\end{abstract}

Copyright () 2019 Institute of Advanced Engineering and Science. All rights reserved.

\section{Corresponding Author:}

Muthanna Jaafar Abbas,

Department of Electrochemical Engineering,

Babylon University,

Hilla, Iraq.

Email: muthab20@gmail.com

\section{INTRODUCTION}

WiMAX technology, the only wireless system which a capable to provide the quality of service (QoS) with a high data rate for IP networks. It can support data rate up to $70 \mathrm{Mbps}$ over $50 \mathrm{~km}$ with support for mobility at car speed. The standard of "Worldwide Interoperability for Microwave Access" product is especially to unmovable and mobile services. Checked to handle full navigation apps. It was absolutely reexamined to cope with the full applications of mobile. Thus, WiMAX can support full mobility for mobile and fixed systems.

The next features provide high information rate; supporting mobile, fixed and peregrine applications, thereby meeting fixed and mobile networks. In additional to the flexibility in network architecture; in summation its cost efficient - which is effective and easy to deploy. They will also give support to "point to multipoint as well point to point connection", additionally give support to IP primarily based architecture; and has enhanced handover which in turn provides support for applications on full mobility i.e. VoIP "Voice over Internet Protocol". It has additionally the energy to save the process which reinforces the battery life of hand-held equipment. "(Internet Protocol Television- IPTV) permits digital tv services for users at a low cost ". Also, IPTV can be a system that is able to obtain a video stream using IP. The mobile users can access IPTV services at anywhere and anytime.

\section{WIMAX TECHNOLOGY ARCHITECTURE}

The WiMAX design was developed with WiMAX support that supports operating, virtual and mobile, in other words, it is a unified network. The WiMAX network framework on the entire IP model. 
There are three major components Consist of them The WiMAX network architecture (Figure 1).

a. (MS): Represents simplicity the user's device which could be set/ mobile and present at the user's location.

b. (ASN): which could be a domain of the WiMAX, that sort the radio access network at the border and it is including one or extra BSs and one or extra ASN gateways.

c. (CSN): One of the main components of the WiMAX which supplies the IP connectivity and everything the IP primary network functions. It also could be termed in the primary network of cellular concept.

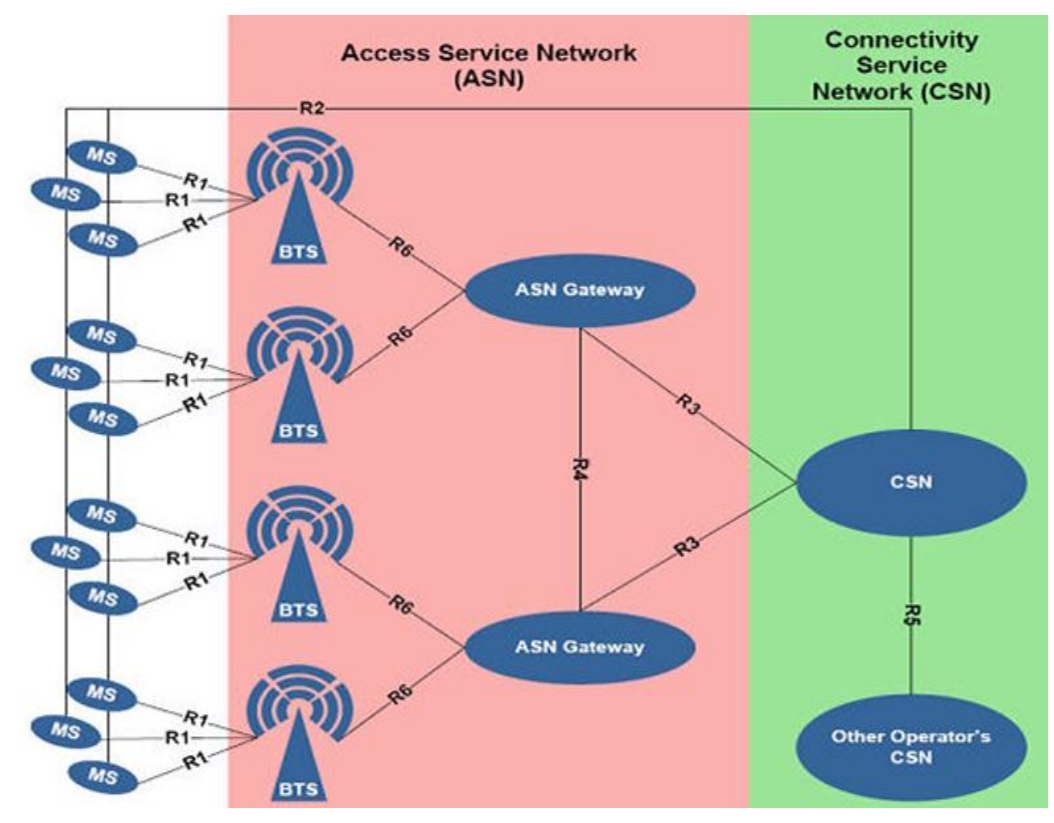

Figure 1. The WiMAX network (reference model)

The overall WiMAX includes "variety" of various entities that structure the various major areas represented higher than those contain the subsequent entities.

a. (SS) and (MS): subscriber station (SS) commonly mentioned as the Client Premises instrumentation (CPE). Later takes spread forms and termed "indoor CPE" or "outdoor CPE", these terms do not need to be explained. The "out of doors CPE" have got the characteristic to provide the best performance because of the best location of the antenna. ( the user may be used the MS), Whereas, the "indoor CPE" is located. These are usually within the variety of an electronic device for a portable computer, etc.

b. (BS): which forms the primary part of a WiMAX network. It's accountable to provide the air interface for the user and mobile stations. In addition to other functions related to the functions of micro-mobility management, such as a hand-off triggering, tunnel foundation, radio resource management, "DHCP (Dynamic Host Control Protocol) proxy", (QoS) Policy Strengthening, Traffic rating, Session management, key and Multicast cluster.

c. (ASN-GW): The ASN gateway in a WiMAX structure is ordinarily a data set node for layer 2 within the total ASN, and could be offer a functions which involve, inner - ASN Site Management transfers and management of radio resources for the temporary storage of a user's profiles and coding key. And also may additionally embody the AAA consumer functionality, institution and management of The possibility of movement and mobility a tunnel with BS, QoS and Policy implementation, Undercover agent functionality for mobile IP, and Routing to the chosen CSN.

d. (HA): it is inside the WiMAX which is specifically located inside the CSN with Mobile-IP forming a key component inside the WiMAX technology. The HA works in combination with a "Foreign Agent", like the ASN gateway to supply an effective end-to-end Mobile IP resolution.

e. Authentication, Authorization and Accounting Server, AAA: in the cellular system and specifically involving within the CSN, the server is used to authenticate the subscription services, accounting and authorization. 


\section{THE QUALITY OF SERVICE IN WiMAX}

Over the past decade, thay have been a significant improvement in the communication networks. Actually, expansion of superior backbone networks was right away followed through the speedy deployment of broadband wireless technologies, similar to leased lines supported fiber-optic links, cable modems utilizing coaxial systems, and (DSL) access networks. This allows the subscriber a whole new category of services by taking advantage of the growing possibilities of the network. A large number of new available services depends on different applications such as (VoIP), (VoD), video conference, large online playing, and P2P, not like traditional TCP/IP services. There are strict warranties for networks like reserved bandwidth or limited delays sometimes needed by multimedia systems applications. QoS means that various things to totally different end users, the maximum amount depends on the appliance and therefore the use to that the top user is putting it. It's so usual to use a variety of measurable performance parameters from that those acceptable to the actual user may be chosen.

\section{SIMULATION ANALYSIS}

The performance of WiMax technology (QPSK and QAM64 modulation techniques) have been analyzed using OPNET Simulator 17.5. In this work, we did analyzing to the impact of Mobility patterns of a mobile WiMax user on VoD through WiMAX. Three mobility patterns were deployed in this Simulation, random nodes, nodes along the track and nodes randomly distributed between fixed and moving. The QAM64 and QPSK technologies have been applied to 7 hexagonal cells in this simulation. The radius of each cell is $28 \mathrm{~km}$. Each cell equipped with one BS which has 16 distributed mobile nodes, with an equal distance between each other and an equal distance from the BS.

\section{RESULTS}

Figure 2 and Figure 3 show the amount of data have been sent for QAM64 and QPSK modulation techniques for time by applying a mode trajectory, fixed and move nodes. Also, A comparison has been made between different Mobility patterns by receive and sent Traffic, individually using QAM64 and QPSK technology.

Figure 2 shows that the throughput the trajectory is higher than random and fixed mobility patterns at the same time.

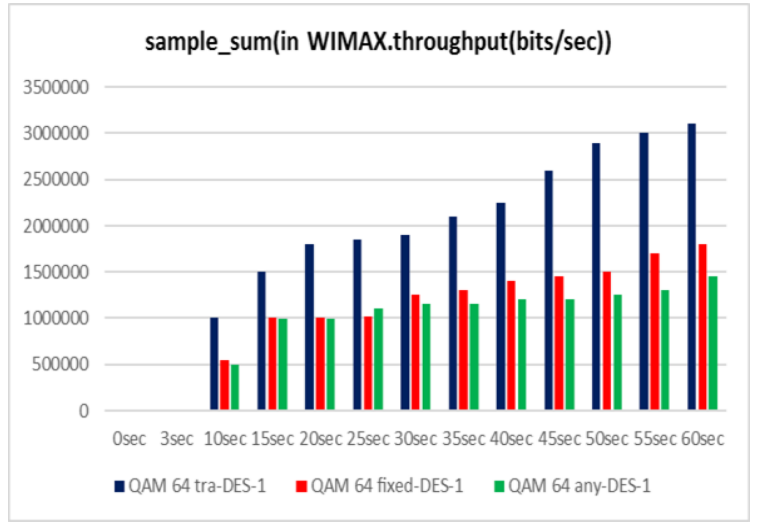

Figure 2. The throughput of QAM modulation technique using different Mobility patterns

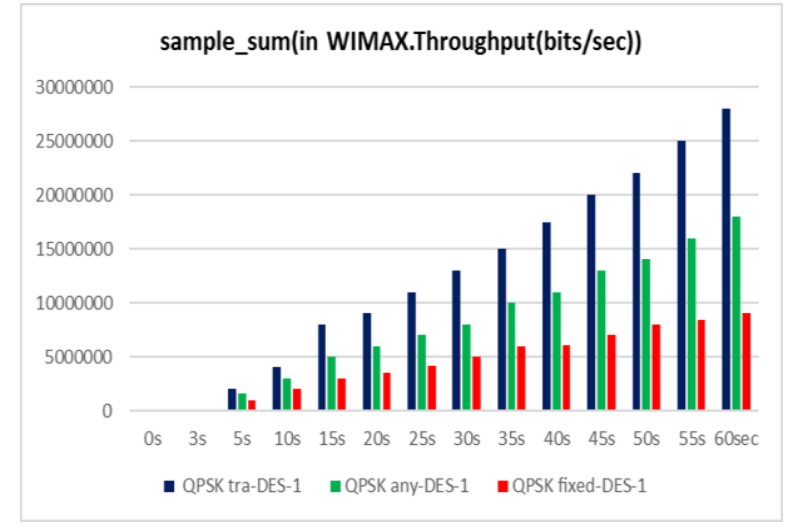

Figure 3. The throughput of QPSK modulation technique using different Mobility patterns

Figure 3 shows that the throughput in the different Mobility Patterns Increasing regularly with a time, the path is higher than from random and fixed mobility patterns respectively. The Figure 4 shows that the levels of Traffic received is higher for the trajectory relative to random and fixed.

The Figure 5 shows that the levels of Traffic received are higher if QPSK modulation technique is used compared to the QAM64, and we can see above that the results exponentially increased proportional for different Mobility patterns. The highest values when the nodes are moving trajectory relative to other mobility patterns. Table 1 shows comparison between QAM64 and QPSK in traffic received in bytes at 30 sec by using different mobility patterns 


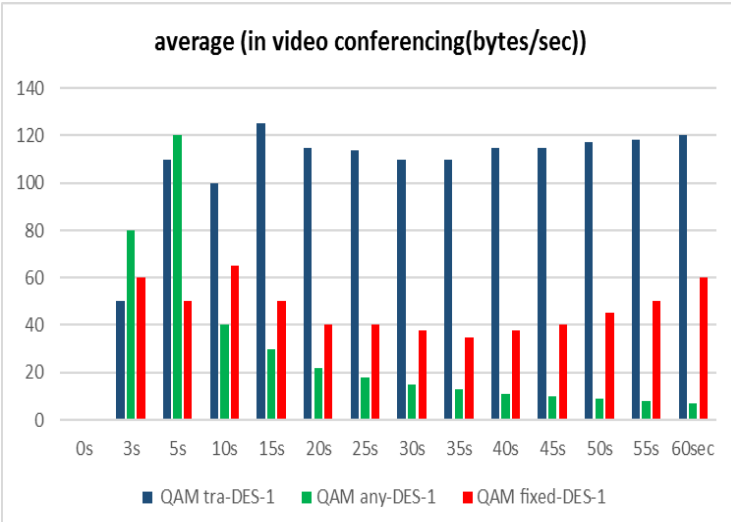

Figure 4. Traffic received of QAM64 modulation technique using different Mobility patterns

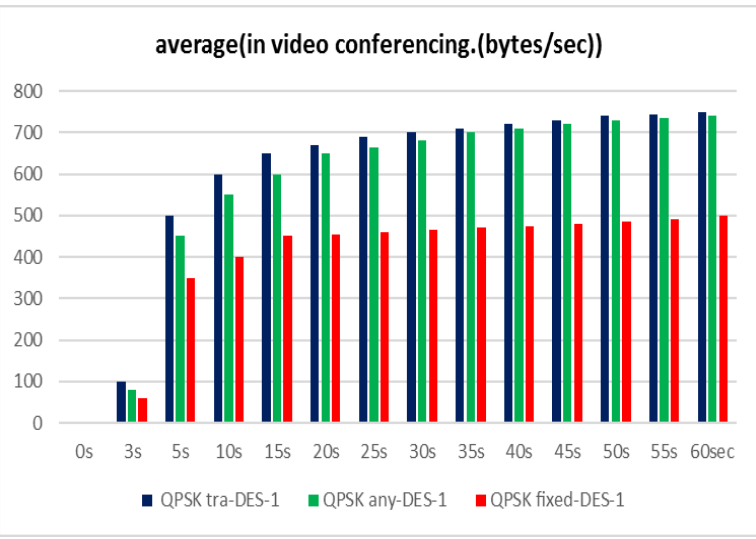

Figure 5. Traffic received of QPSK modulation technique using different Mobility patterns

Table 1. Comparison between QAM64 and QPSK show Traffic received in bytes at 30 sec by using different Mobility patterns

\begin{tabular}{cccc}
\hline & Tra & any & fixed \\
\hline QAM64 & 110 & 17 & 38 \\
QPSK & 700 & 680 & 470 \\
\hline
\end{tabular}

The Figure 6 shows that levels Traffic sent be higher for the trajectory relative to random and fixed. For example, after 30 seconds, we can see that when the nodes are moving anywhere the Traffic sent value is 4900000 Compare with values 3100000 and 1800000 for fixed and trajectory Respectively.

The Figure 7 shows that the levels of Traffic sent is higher for the trajectory relative to other Mobility patterns. For example, after 30 seconds, we can see that when the nodes are moving in a trajectory the Traffic sent value became 63000 compare with the values of 43000 and 29000 for anywhere and fixed respectively.

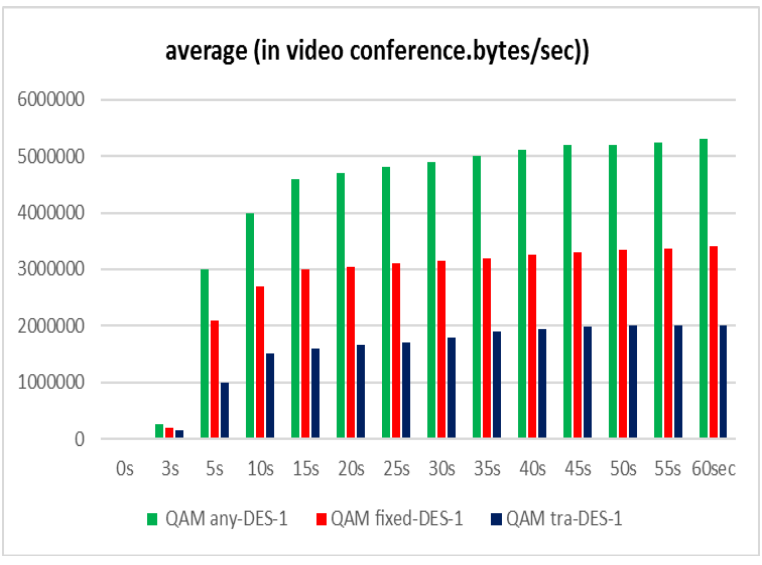

Figure 6. Traffic sent for QAM64 modulation techniques by using different Mobility patterns

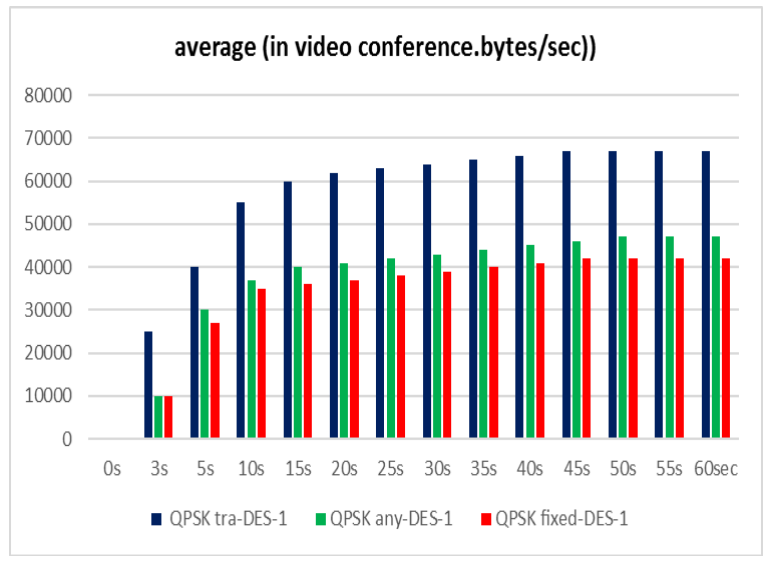

Figure 7. Traffic sent for QPSK modulation techniques by using different Mobility patterns

In Table 2 we can see the comparison between QAM64 and QPSK show traffic sent in bytes at 30 sec by using different mobility patterns. 
Table 2. Comparison between QAM64 and QPSK show Traffic sent in bytes at $30 \mathrm{sec}$ by using different Mobility patterns

\begin{tabular}{cccc}
\hline & Tra & any & fixed \\
\hline QAM64 & 1850000 & 4950000 & 3150000 \\
QPSK & 640000 & 44000 & 39000 \\
\hline
\end{tabular}

\section{CONCLUSION}

We deployed QPSK and QAM64 modulation techniques in (WiMAX) a network for different Mobility Patterns to transfer video streaming. The target of this paper is the QoS over WiMAX access technology. The result is an effective and attainable overall the performance of (QPSK) modulation technique compared to (QAM) modulation. Generally, the best results we got it in this work, when the mobile node is moving. In the future, we plan to work with the possibility of implementing data transmission networks and through the transfer of more data capacity, more coverage space and a larger number of users and different modes of navigation and specific terrain, we can discuss our next paper.

\section{REFERENCES}

[1] http://whirlpool.net.au/wiki/?tag=VoIP_Codecs.

[2] F. Ehtisham, et al., "Performance Evaluation of Secure Video Transmission over WiMAX," International Journal of Computer Networks \& Communications (IJCNC), vol/issue: 3(6), 2011.

[3] J. Hamodi, et al., "Evaluating the Performance of IPTV over Fixed WiMAX," International Journal of Computer Applications, vol/issue: 84(6), 2013.

[4] Priyanka and J. Malhotra, "Performance Evaluation of Scheduling Services for VoIP in WiMAX Networks," International Journal of Computer Applications, vol/issue: 71(19), 2013.

[5] Anouari, et al., "Performance Analysis of VoIP Traffic in WiMAX using various Service Classes," arXiv preprint arXiv:1308.0223, 2013.

[6] F. Zivkovic, et al., "Quantitative Analysis of Streaming Multimedia over WiMAX and LTE Networks Using OPNET v. 16.0," Group, 2013.

[7] J. George and P. P. Winston, "Fast Mobile WiMAX handover by optimizing network topology acquisition phase procedure," Circuit, Power and Computing Technologies (ICCPCT), 2014 International Conference on, pp. 16821686, 2014.

[8] K. Jaswal, et al., "OPNET Based Simulation and Investigation of WiMAX Network Using Different QoS," International Journal of Research in Engineering and Technology (IJRET), pp. 575-579, 2014.

[9] S. Sharma, et al., "A Review on Quality of Services Scheduling Algorithms in WiMAX," 2016.

[10] A. Kaur, et al., "Performance comparison of wimax classesover voip," International Journal for Science, Management and Technology (IJSMT), vol/issue: 6(6), 2016.

[11] Z. Li and J. Yang, "Investigation on Handover in WiMAX and Performance Comparison over WiMAX and LTE," 2016.

[12] EXata documentation. http://www.scalablenetworks.com

[13] H. M. Hathal, "Performance Evaluation of Different Modulation Coding for Scheduling Services over VoIP in WIMAX Networks," SAUSSUREA, vol/issue: 6(1), pp. 27-33, 2016.

[14] M. M. A. Eljalil and A. B. Abdelnabi, "Quality of Service for Real Time Video Transferring in Wireless WiMAX Technology," International Journal of Computer Science and Mobile Computing (IJCSMC), vol/issue: 6(10), pp. 74-82, 2017.

[15] B. M. AL-Mahadeen and A. Al-Mseden, "Improving the QoS of VoIP over WiMAX Networks Using OPNET Modeler," International Journal of Computer Science and Network Security, vol/issue: 17(8), 2017.

[16] https://www.ijcaonline.org/ 